\title{
Influencers on YouTube: a quantitative study on young people's use and perception of videos about political and societal topics
}

\author{
Daniel Zimmermann ${ }^{1}$ (1) $\cdot$ Christian Noll ${ }^{2}$ Lars Gräßer ${ }^{3} \cdot$ Kai-Uwe Hugger ${ }^{2} \cdot$ Lea Marie Braun ${ }^{2} \cdot$ Tine Nowak $^{2}$. \\ Kai Kaspar ${ }^{1}$
}

Accepted: 30 October 2020 / Published online: 18 November 2020

(C) The Author(s) 2020

\begin{abstract}
The roles of YouTube videos and YouTubers for getting information about political and societal topics are becoming gradually more important to young people. Quantitative research about young people's use and perception of YouTube-videos and their potential effects on opinion formation is sparse though. This cross-sectional quantitative study addresses this empirical gap. We examined young people's analytic-critical evaluations of YouTubers and their videos about political and societal topics (YTPSvideos), and how these are affected by the young people's age and gender. We analysed questionnaire data of 562 participants and divided them into three different age groups. Overall, the participants reported a moderate watching frequency of YTPS-videos. They also rated YTPS-videos as moderately credible and considered specific characteristics of YTPS-videos and their producers as being moderately indicative for fake news. When comparing to traditional TV news, YTPS-videos were perceived as more entertaining, emotional, funny, exciting, modern, and motivating but also as more subjective and manipulating. Regarding YouTubers, the participants ascribed them an important role model function, but criticised them for handling it rather irresponsibly. Concerning opinion formation processes, the participants reported of a rather unimportant role of YTPS-videos for their learning about political and societal topics. They also perceived themselves as less influenceable than other peers and younger people. Still, they prefer talking with friends instead of teachers about YTPS-videos. Age and gender also had effects on various scales. These results deliver essential data for future research and educational measures and opened up unexplored areas in this research field.
\end{abstract}

Keywords YouTube $\cdot$ Politics $\cdot$ Fake news $\cdot$ News perception $\cdot$ Gender differences $\cdot$ Age differences

\section{Introduction}

Social media is an important information source for young people (Gangadharbatla, Bright, \& Logan, 2014; Kaspar \& MüllerJensen, 2019), and YouTube seems to be especially relevant. Young people consume YouTube-videos to obtain new information and forming their own opinion (Schmidt, Hölig, Merten, \& Hasebrink, 2017). In this context, the role of YouTube creators and their videos on political and societal topics become gradually more important (Lewis, 2020). However, research

Daniel Zimmermann

d.zimmermann@uni-koeln.de

1 Department of Psychology, University of Cologne, Richard-Strauss-Str. 2, 50931 Cologne, Germany

2 Department of Educational and Social Sciences, University of Cologne, Gronewaldstr. 2, 50931 Cologne, Germany

3 Grimme-Institut, Eduard-Weitsch-Weg 25, 45768 Marl, Germany on how young people use and perceive such videos and how they perceive the potential effects of such videos on their opinion formation is sparse, primarily quantitative approaches. The present study aims to fill this empirical gap by exploring (1) how intensively young people watch YouTuber videos about political and societal topics (YTPS-videos), (2) how young people evaluate YouTube creators (YouTubers) and the content of their videos, (3) how young people perceive their influenceability by YTPS-videos, (4) with whom and via which channels they communicate about the content of YTPS-videos, and (5) how these aspects are affected by young people's age and gender. Importantly, in the present study, YTPS-videos are not restricted to a specific format (e.g., commentary, interview, report) and cover any kind of news about political or societal events being of potential interest to the audience.

\section{Intensity of YouTube Usage}

In April 2020, YouTube had the second-highest number of active users worldwide after Facebook, making it the most 
popular and most important online video platform to date (Statista, 2020). Nearly two-thirds of 12- to 19-year-old German teenagers indicated YouTube as their favourite online platform in 2019 (MPFS, 2020), with 90\% of them watched videos on YouTube regularly. Similar results were found in Britain (Jiménez, García, \& de Ayala, 2016) and Israel (Zilka, 2018). Also, Zilka (2018) found significant gender and age effects regarding preferred media content. Consequently, and concerning the present study, the question arises how intensively young people watch YTPS-videos and how this is related to their general YouTube usage intensity, visiting frequency of YouTuber channels as well as gender and age:

RQ1. How frequently do young people of different age and gender watch YouTube and how intensively do they watch YTPS-videos in particular?

\section{Evaluation of YouTubers and YTPS-Videos}

When it comes to the evaluation of YouTubers and the content of YTPS-videos, credibility is one central aspect. Beyond a positive relationship between the perception of media credibility and news consumption (Nelson \& Kim, 2020), credibility affects the persuasiveness of media content. The Source Credibility Model (cf. Lowry, Wilson, \& Haig, 2013) emphasises that the source of a message and its perceived expertise and trustworthiness are determinants of the message's persuasiveness and perceived usefulness, as shown for various scenarios in the context of social media (e.g., Djafarova \& Rushworth, 2017; Lou \& Yuan, 2019). Besides, credibility was also found to be positively related to the general liking of YouTuber videos (Xiao, Wang, \& Chan-Olmsted, 2018). Moreover, the evaluation of the credibility of online information depends on the recipient's age. For example, college students perceived online information as more credible and verified them more rarely than older adults did (Metzger, Flanagin, \& Zwarun, 2003). Hence, we examined the following research question concerning YTPS-videos:

RQ2a. How do young people of different age and gender evaluate YTPS-videos' credibility, how much do they like these videos, and how are these variables correlated with watching frequency?

The Source Credibility Model stresses the importance of the message's source for its persuasiveness. However, the 'source' can refer to both the video and the YouTuber who produces and promotes the video. The question arises which characteristics of YTPS-videos and YouTubers young people use when evaluating credibility aspects and when checking for fake news. This question is particularly important in the context of videos about political and societal topics. Some studies have already examined (non-)credibility in terms of fake news during periods of a political election. Grinberg, Joseph, Friedland, Swire-Thompson, and Lazer (2019) and Guess, Nagler, and Tucker (2019) examined the sharing of and exposure to fake news on Twitter and Facebook, respectively, during the 2016 United States presidential campaign. The authors found that mostly conservative, older people were most likely to be engaged in fake news sources.

When it comes to the detection of fake news on social media, previous research often examined characteristics of news content which might be indicative of fake news, such as linguistic- and visual-based content features of news (Shu, Sliva, Wang, Tang, \& Liu, 2017). However, young people's strategy to detect fake news is still unclear. A first qualitative study delivered some hints: Hugger et al. (2019) found that young people's credibility evaluation of the content of YTPSvideos appears to be strongly interlinked with perceived authenticity of the YouTubers; the perceived authenticity seems to be promoted by perceived individuality and autonomy of the YouTuber but is compromised by an increasing professionality of editorial work and news presentation. The present study hence further explored the characteristics of YTPS-videos that young people use to assess the news' validity:

RQ2b. Which characteristics of YTPS-videos do young people of different age and gender consider indicative of fake news?

Additionally, young people seem to use traditional TV news as a reference to evaluate the credibility and the news value of YTPS-videos. Hugger et al. (2019) found that in order to assess the credibility of YouTubers and YTPS-videos, young people at the age of 15 to 16 reported that they compare YouTube with traditional mass media such as TV news, which are perceived as more objective and reliable. The authors speculate that this result may indicate a relatively low ability of young adolescents to analyse and reflect media content critically. In contrast, young adults explicitly perceived the mixing of personal opinions and objective information in mass media more strongly, indicating a significant role of the user's age on media criticism. Though, it is unclear how this result pattern is quantitatively represented:

RQ2c. How do young people of different age and gender evaluate YTPS-videos compared to traditional TV news?

Besides the video itself, its producer plays a vital role as an information source. Recent research supports this claim: Lou and Yuan (2019) found influencer's trustworthiness, attractiveness, and similarity to their followers being factors that positively affect followers' trust in the messages and 
postings. Xiao et al. (2018) found that the trustworthiness and the social influence of YouTubers affect the perceived information credibility on YouTube. Furthermore, Balaban and Mustățea (2019) showed that attractiveness, trustworthiness, expertise, and similarity are essential factors affecting the perceived information credibility, at least in Romania and Germany. The evaluation of these factors may be additionally moderated by the gender of both the media user and the influencer (Todd \& Melancon, 2018).

Moreover, recent studies shifted their focus to the perceived role model function of influencers. To put it briefly, recipients tend to identify more with, feel more similar to, and trust influencers more than celebrities (Schouten, Janssen, \& Verspaget, 2020). In a recent literature review, De Veirman, Hudders, and Nelson (2019) stressed the importance of influencers as relatable and approachable role models, as children are willing to build (parasocial) relationships and to identify with them, which in turn can lead to the adoption of the influencers' behaviour and opinion. In contrast, Martínez and Olsson (2019) showed in their qualitative study that children are capable of critically reflecting upon YouTubers, regarding their celebrity status and commercialisation especially. Interestingly, the degree of reflexivity differed between children. Thus, children do not seem to be a homogenous group when it comes to critical perceptions of YouTubers. Moreover, young people and women tend to have more trust in other people on social media, as compared to older people and men, respectively (Warner-Søderholm et al., 2018). Consequently, with regard to young people's capability of media criticism in the context of YTPS-videos, we asked the following question:

RQ2d. To what extent do young people of different age and gender perceive YouTubers as reliable and responsible role models?

\section{Learning with and Perceived Influenceability by YTPS- Videos}

According to the core idea of uses-and-gratification models, people use media to satisfy particular needs such as the need for information and they hence actively link need gratification to media choice. Kaspar and Müller-Jensen (2019) summarised that uses-and-gratification models "usually assume that media recipients have certain psychological and societal needs (and associated motives), eliciting specific expectations about how mass media can fulfil these needs and leading to corresponding behavioural patterns of media use in order to obtain the desired gratifications at the end" (p. 2). So, it can be assumed that users might watch YTPS-videos specifically for acquiring new information and expand contentspecific knowledge. Several studies highlighted the use of
YouTube for learning purposes concerning students' academic learning (Moghavvemi, Sulaiman, Jaafar, \& Kasem, 2018), teenagers' knowledge and skill acquisition (Pires, Masanet, \& Scolari, 2019), and as an informal learning tool for children (Dyosi \& Hattingh, 2018). Use motives also seem to differ between genders, with men showing more informationseeking intentions when using social networks than women (Krasnova, Veltri, Eling, \& Buxmann, 2017). Accordingly, we examined the following research question:

RQ3a. How important do young people of different age and gender perceive YTPS-videos for knowledge acquisition about politics and societal topics?

In addition to the purposeful and intentional use of YouTube for knowledge acquisition (RQ3a), it is unclear as yet how young people assess the general potential of YTPSvideos to (unintentionally) influence their opinion formation. In recent years, a similar realm of this process was frequently examined under the terms of dispositional and situational advertising literacy in the field of marketing (Hudders, Cauberghe, \& Panic, 2016): The ability to critically reflect upon advertising depends on the recipients' coping skills. These skills thereby include recognising, analysing, and evaluating persuasion attempts as well as choosing an effective coping strategy against them. Interestingly, media literacy seems to improve with age (De Jans, Hudders, \& Cauberghe, 2018), because older children (ten to eleven years) are better in detecting commercial attempts and brand placements than younger children (seven to eight years) (Hudders \& Cauberghe, 2018). Besides, eight- to twelve-year-old children did not show the same level of media literacy as 18- to 30-year-old adults (Rozendaal, Buijzen, \& Valkenburg, 2010). In contrast, results of previous studies show that even older adolescents show engagement in the activities of influencers instead of critically reflecting upon them (van Dam \& van Reijmersdal, 2019). However, they perceive themselves as capable of understanding advertising and being somewhat resistant to it (De Jans et al., 2018).

Furthermore, psychological research in several domains revealed that one's influenceability by mass media is often perceived as less pronounced than the influenceability of other peers. This phenomenon has been labelled the thirdperson effect in communication (Davison, 1983). The third-person effect describes the persistent phenomenon that people perceive communication effects on others as stronger than on oneself. This phenomenon applies to a variety of communication forms, including social media effects and online news perception (e.g., Banning \& Sweetser, 2007; Houston, Hansen, \& Nisbett, 2011; Schweisberger, Billinson, \& Chock, 2014). We thus expected similar results with respect to YTPS-videos and explored the following question: 
RQ3b. How strongly do young people of different age and gender perceive their own as well as other young people's influenceability by the content of YTPSvideos?

\section{Communication about the Content of YTPS-Videos}

Finally, seeking the advice of others is a vital strategy to verify, evaluate and validate online information (Metzger et al., 2003). People use interpersonal communication via social media to confirm their own opinion, to obtain personal recommendations, or to orientate themselves by feedback systems (Metzger, Flanagin, \& Medders, 2010). Thereby, some sources are perceived as more trustworthy than others: People prefer the recommendations and opinions of friends and family. Indeed, online comments of users affect news processing and credibility perception of news content and sources (Duong, Nguyen, \& Vu, 2020; Waddell, 2018a, $2018 b$ ). Referring to the communication channels, the use of a particular communication channel apparently depends on the type of friend (strong versus weak ties) and the communication subject (school subject versus no school subject) (Van Cleemput, 2010). In line with that, when sharing emotions, Flemish adolescents were found to prefer talking face-to-face, followed by texting, calling, and posting on social networks (Vermeulen, Vandebosch, \& Heirman, 2018). Additionally, males seem to tend to communicate more frequently via public comments on YouTube videos but show less video-sharing behaviour with others than females do (Khan, 2017). Therefore, concerning YTPS-videos, we examined with whom and via which channels young people of different age and gender talk about the content of such videos.

RQ4a. With whom do young people of different age and gender communicate about the content of YTPSvideos?

RQ4b. Which channels do young people of different age and gender use to communicate with others about YTPSvideos?

\section{Methods}

\section{Sample}

We created both a pencil-paper and an online version of the survey with the software Unipark (Questback, 2017). The pencil-paper version was distributed among pupils of cooperating schools and students of a large German university. Written informed consent to participate was provided by the pupils' legal guardians/next of kin, and all of them participated voluntarily in this study in the context of formal school teaching. An online version of the questionnaire was additionally distributed via specific participant recruitment groups on Facebook in order to reach groups of older people with a serious interest in participating in scientific research. After clicking on the link to open the study, we pointed out that participation in the online survey required a minimum age of 16 years, following the guidelines of the General Data Protection Regulation of the European Parliament (GDPR, 2016). We also informed the participants that they could stop the study whenever they want, and that completion of the survey would indicate informed consent. We did not collect any identifying personal data.

Overall 739 participants (577 pencil-paper, 162 online) took part in the survey. We initially excluded 135 participants (18.27\%) from the analyses as they reported neither using YouTube in general nor consuming YouTuber videos about political or societal issues (YTPS-videos), further 7 participants $(0.95 \%)$ for not completing the survey, further 11 participants $(1.49 \%)$ due to missing data regarding age or gender, further 9 participants $(1.22 \%)$ for defining their gender as 'others', which was an insufficient subsample for the gender-related statistical analyses, and further 15 participants ( $2.03 \%$ ) for reporting being older than 35 years. Therefore, the data of 562 participants (440 pencil-paper, 122 online) with $59.07 \%$ females were included in the analyses. The mean age was $M_{\text {age }}=18.87\left(S D_{\text {age }}=4.86\right)$. The number of subscribed YouTube channels reported by these participants showed a median of 10 . At the time of the survey, $55.0 \%$ of the participants were pupils of secondary schools, $40.2 \%$ were students of a University, and $4.8 \%$ attended neither of these. We divided participants into three different age groups to examine age effects: Participants who were under the age of 16 and therefore not authorized by law to vote in Germany $(n=205$, $48.29 \%$ female; $M_{\text {age }}=14.39, S D_{\text {age }}=0.64$ ), young people from 16 to 20 years of age $\left(n=156,62.18 \%\right.$ female; $M_{\text {age }}=$ $\left.17.64, S D_{\text {age }}=1.66\right)$, and participants older than 20 years of age (range from 21 to 35 years) and therefore legally get full criminal responsibility as an adult in Germany $(n=201$, $67.66 \%$ female; $\left.M_{\text {age }}=24.39, S D_{\text {age }}=3.34\right)$. The number of participants slightly varied across the analyses due to occasional missing data.

\section{Questionnaire}

The introduction of the survey included our definitions of the terms "YouTuber" and their "YTPS-videos". We defined YouTubers as people who run their own YouTube channel and upload regularly or irregularly self-produced videos on YouTube. Thus, we clarified that the survey is not about videos produced by professional news broadcasters that additionally disseminate their news via YouTube. We defined 
YTPS-videos as videos of any kind of format addressing political or societal topics.

We initially asked the participants to indicate a few demographical information: age, gender, school, previous graduation (if they already had one), and their occupational status (if they were not attending any school). If not stated otherwise, each of the following questions was answered on a 5-point rating scale ranging from 1 ("not at all" or "never") to 5 ("very much" or "very often").

First, we assessed participants' YouTube usage intensity. We asked them how intensively they use YouTube in general, how frequently they watch YTPS-videos, and how frequently they visit YouTuber channels on a 6-point scale $(1=$ "every day", 2 = "every week, but not every day", 3 = "every month, but not every week", 4 = "every year, but not every month", 5 = "less than once a year", $6=$ "never"), see Table 1 .

Second, we assessed participants' evaluation of the credibility of YouTubers and their videos. As shown in Table 1, we asked them to estimate the credibility of YTPS-videos in general, how much they appreciate it when YouTubers present their own opinion in their YTPS-videos, and to evaluate their personal liking of YTPS-videos in general $(-2=$ "very negative", $0=$ "neutral", $+2=$ "very positive"). Furthermore, the participants rated several characteristics of YouTubers and their videos they potentially regard as indicative of Fake News ( 1 = "no indicator at all", $3=$ "medium indicator", $5=$ "very strong indicator"). The full list of evaluated characteristics can be seen in Table 1.

Third, participants compared YTPS-videos with classic TV news. To do so, they assessed 20 characteristics whether these apply rather to YTPS-videos or to TV news $(1=$ "applies much more to YTPS-videos", 3 = "applies equally to both formats", 5 = "applies much more to TV news"). Importantly, numeric markers were introduced for the analyses but were not shown to the participants in order not to bias their ratings. We only presented the verbal markers to the participants. The full list of evaluated characteristics is presented in Table 2.

Fourth, we assessed participants' learning and perceived influenceability by YTPS-videos. On the one hand, participants rated the importance of YTPS-videos for their learning about politics and societal topics. We asked them how important YTPS-videos are to them as a source of information, how much they learn by watching such videos, and how strongly they use the content of YTPS-videos for their opinion formation. We calculated a mean score across these items (Cronbach's $\alpha=.811$ ). On the other hand, participants assessed how strongly they let themselves be influenced by YTPS-videos and to what extent other people of the same age, younger people, and older people are influenced by such videos.

Fifth, we assessed the extent to which YouTubers are perceived as role models. Participants rated several items regarding their perception of YouTubers' role model function. The full list of items is presented in Table 3.

Finally, we examined participants' communication about the content of YTPS-videos. Participants rated the frequency of talking with certain communication partners and the communication channels they use. The full list of communication partners and channels is presented in Table 4 .

\section{Analyses}

All analyses were conducted using SPSS 27. In order to quantify the extent of participants' evaluations of YouTubers and YTPS-videos as below-average, average, or above-average compared to the scale's midpoint, we conducted one-sample $t$-tests. We analysed possible main and interaction effects of age group $(<16$ years, $16-20$ years, $>20)$ and gender using $3 \times 2$ ANOVAs. In case of a significant main effect of age group, we performed pairwise comparisons (Bonferronicorrected familywise error rate). All tables display the means and standard deviations for age and gender groups, indicate the results of the one-sample $t$-tests, and present the results of the ANOVAs including pairwise comparisons for age groups. Moreover, due to multiple testing and accumulated Type I error, we further report Bonferroni-adjusted significance levels for each set of $t$-tests and each set of ANOVAs associated with a research question. However, due to the explorative nature of the present study and because several authors have already pointed out that the Bonferroni method is unnecessarily conservative in case of many tests and hence increases the false-negative rate (e.g., Jafari \& Ansari-Pour, 2019; Lee \& Lee, 2018), we also discuss effects being significant only in the case of unadjusted significance levels. As the results are the first of its kind, applying a stricter criterion could otherwise obscure essential effects. Nevertheless, we additionally indicate the more conservative (adjusted) significance levels in the tables and figure for transparency reasons.

\section{Results}

\section{YouTube Watching Habits (RQ1)}

As shown by Table 1, the general YouTube usage intensity was consistently rated as above average across age groups and genders. Visiting frequency of YouTuber channels was consistently under the scale's midpoint, indicating an aboveaverage frequency due to reversed item coding $(1=$ "every day", $6=$ "never"). The watching frequency of YTPS-videos was stated to be either average or below average across groups. Besides, males reported a higher general usage intensity of YouTube, a higher visiting frequency of YouTube channels, and a higher watching frequency of YTPS-videos. The 16- to 20-year-old participants stated a higher usage 


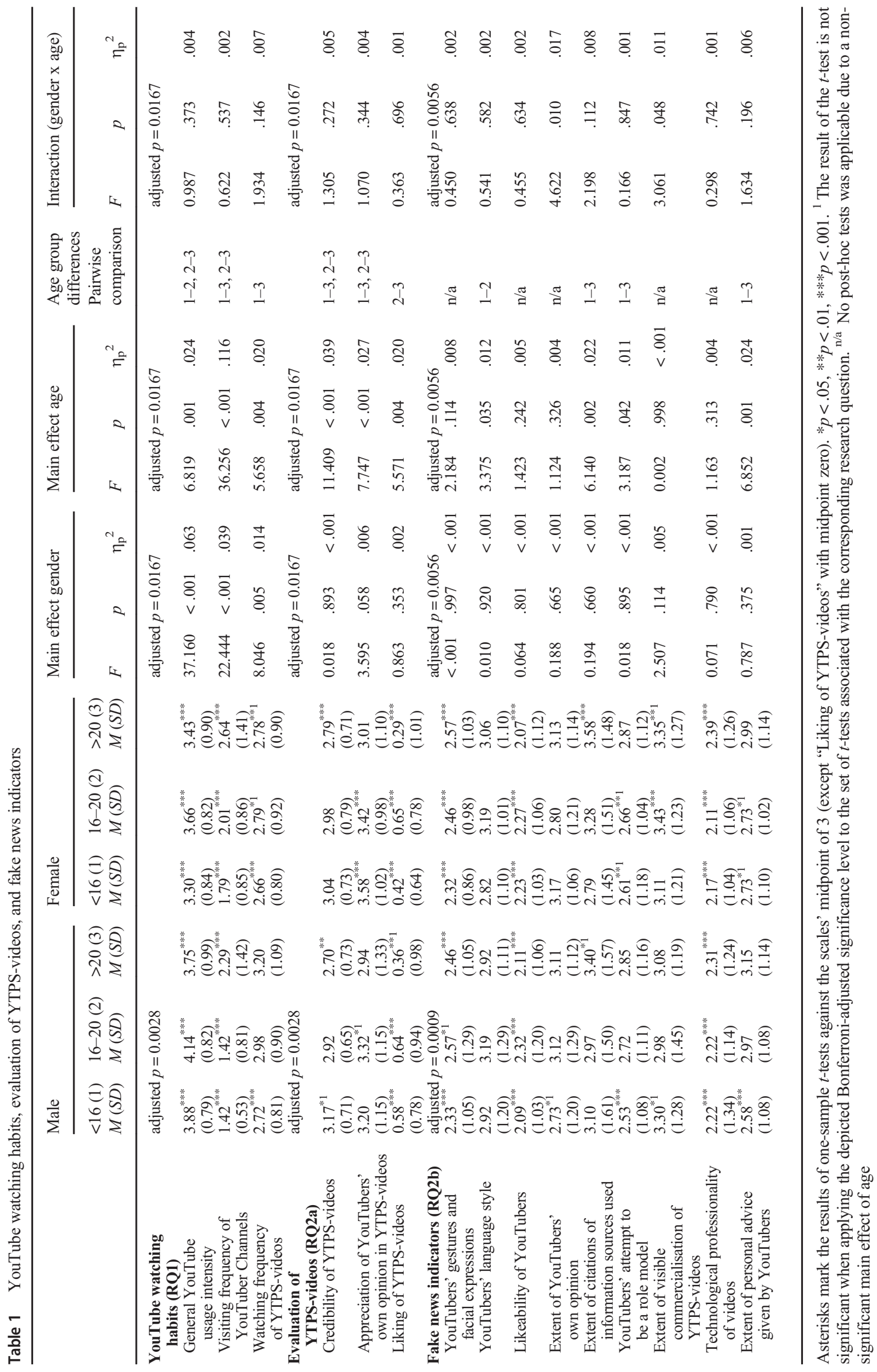









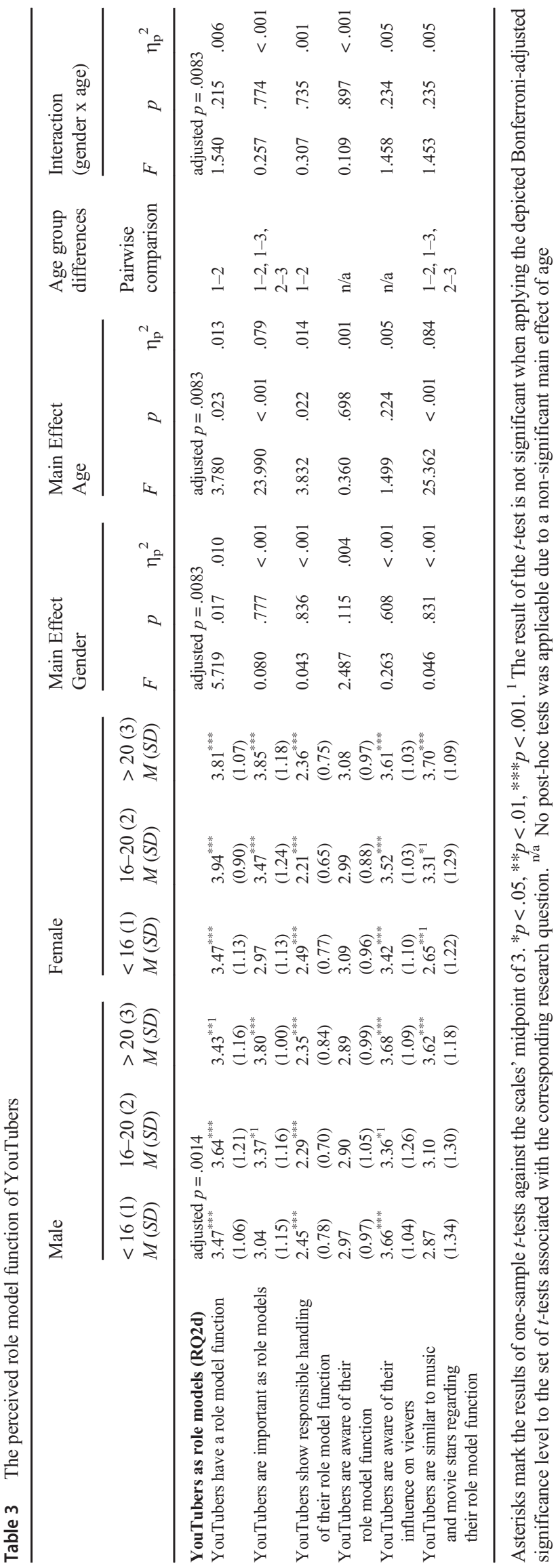

intensity of YouTube compared to the other two age groups. The over-20-years-old group stated a lower visiting frequency of YouTuber channels than the other two groups (reversed coding) but reported to watch YTPS-videos more frequently than the under-16-year-old group. Since the scale measuring the visiting frequency of YouTuber channels was rather ordinal level, we also performed non-parametric analyses that verified the parametric results reported in Table 1.

\section{Evaluation of YTPS-Videos and Fake News Indicators (RQ2a, RQ2b)}

Only the under-16-year-old males evaluated the credibility of YTPS-videos above average (see Table 1), whereas each of the other groups rated the credibility as either average or below average (RQ2a). The appreciation of the presentation of YouTubers' own opinion in YTPS-videos was average or above average, while the liking of YTPS-videos was rated as above average (scale's midpoint was zero). The over-20-yearold evaluated YTPS-videos as less credible and reported a lower appreciation of YouTubers' own opinion than both younger age groups. Further, they stated a lower liking of YTPS-videos than the 16- to 20-year-old group. There was no effect of gender. Additionally, we found significant positive correlations between the credibility evaluation and the watching frequency of YTPS-videos for over-20-year-old males, $r=.283, p=.023$, and females, $r=.287, p<.001$. There were no significant correlations in the other age groups regardless of gender, all $r \mathrm{~s} \leq .194, p \mathrm{~s} \geq .057$. Besides, we found significant positive correlations between the credibility evaluation and the appreciation of the presentation of YouTubers' own opinion in all groups, with the highest values for over-20-year-old males, $r=.406, p<.001$, and females, $r=.331, p<.001$. Except for 16 - to 20 -year-old males, we also found significant positive correlations between the credibility evaluation and liking of YTPS-videos in all groups, with the highest expression for over-20-year-old males, $r=.374, p=.002$, and females, $r=.480, p<.001$.

Regarding characteristics of YouTubers and their videos considered as potentially indicative of fake news (RQ2b), most mean ratings were average or below average (see Table 1). As an exception, the citation of information sources was considered as indicative above average by over- 20 -year-old males and females. The extent of visible commercialisation of YTPS-videos was considered as indicative above average by under-16-year-old males as well as by 16 - to 20 -year-old and over-20-year-old females. The ANOVAs did not find differences between males and females, but some main effects of age group: The under-16-year-old group rated the YouTubers' language style as a less strong indicator than the 16- to 20-yearold group. Concerning the citation of information sources, YouTubers' attempt to be a role model, and the extent of personal advice given by the YouTubers, the under-16-year-old 


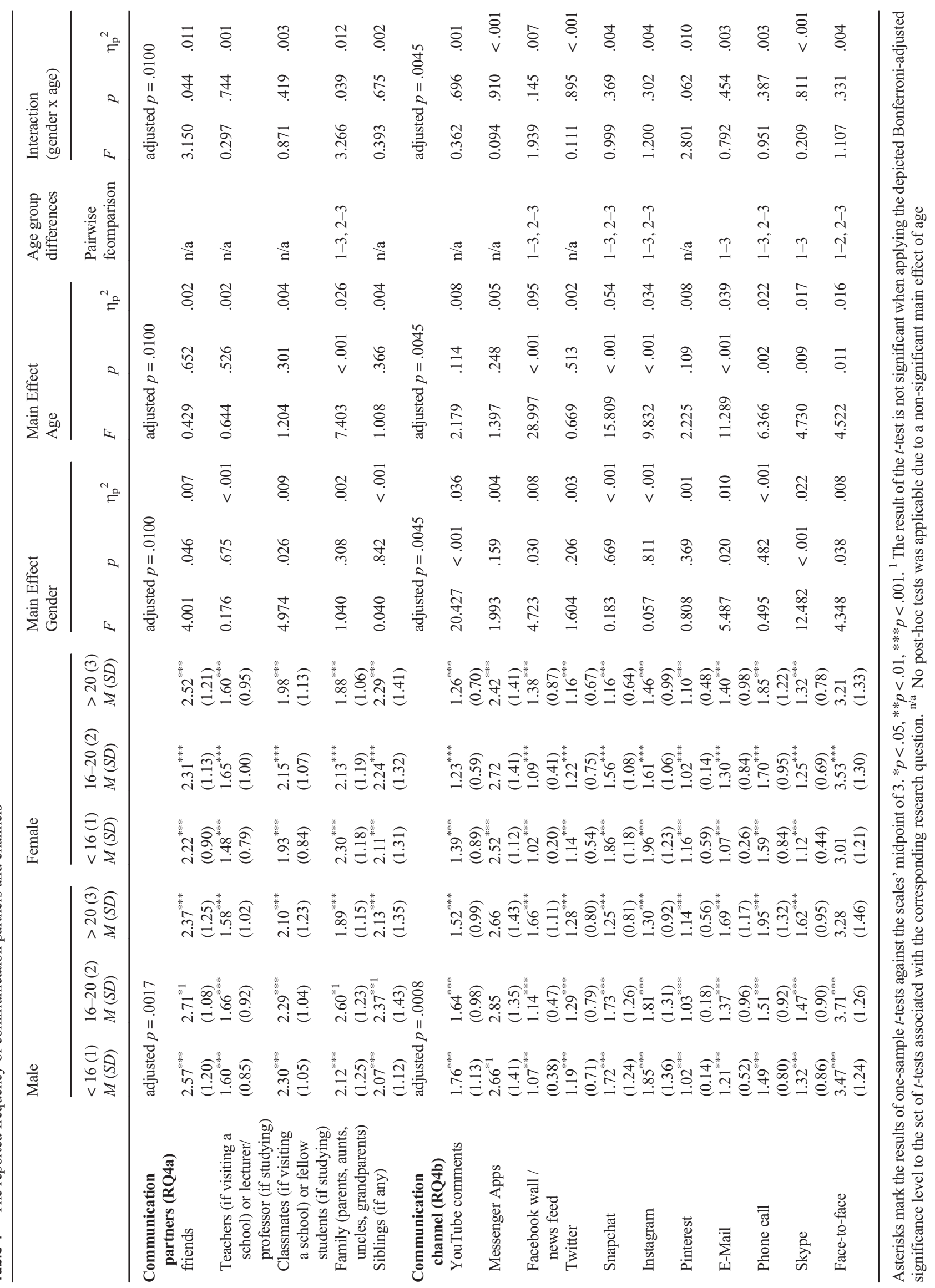


group perceived them as less strong indicators of fake news than the over-20-year-old group did. Furthermore, we found an interaction between age and gender regarding the extent to which YouTubers' express their own opinion in YTPS-videos: Females showed a higher rating than males in the group of the under-16-year-old group, $t(202)=2.76, p=.006, d=0.386$, whereas no gender difference was found in the other two age groups. There was also an interaction effect between age and gender relating to the extent of visible commercialisation in YTPS-videos, as females showed a higher rating than males only in the 16- to 20-year-old group, $t(149)=2.03, p=.044$, $d=0.343$.

\section{YTPS-Videos Versus TV News (RQ2c)}

Participants rated whether several characteristics apply rather to YTPS-videos or traditional TV news (RQ2c), see Table 2. Ratings that significantly differed from 3 indicate that the corresponding characteristic was attributed more to YouTuber news (below 3) or more to TV news (above 3). Overall, TV news was seen as more objective, facts-oriented, informative, boring, credible, neutral, reputable, and professional, independently of participants' age and gender. In contrast, YTPS-videos were generally seen as more subjective, opinion-oriented, entertaining, emotional, funny, exciting, modern, motivating, and manipulative. Both formats were rated as equally interesting. The characteristics "compact" and "exhausting" were equally attributed to both news formats or rather to TV news, depending on age and gender. Moreover, the ANOVAs revealed some main effects of gender and age group: In contrast to male participants, female participants rated TV news as more objective, facts-oriented, informative, exciting, credible, neutral, reputable, and compact, but they rated YTPS-videos as more subjective, opinion-oriented, emotional and manipulative. Regarding age, the over-20year-old group rated YTPS-videos as less opinion-oriented, entertaining, and motivating than the other two age groups, but they also rated TV news as less neutral. Besides, they also perceived YTPS-videos as less exciting than the 16-20-yearold group, and as less manipulative than the under-16-year-old group. At last, they rated TV news as less exhausting compared to the under-16-year-old group. There was no interaction effect between age and gender on any of the characteristic ratings.

\section{YouTubers as Role Models (RQ2d)}

According to participants' perception, YouTubers have a role model function, are important as role models, are aware of their role model function and their influence on viewers, and they are similar to music and movie stars regarding their role model function (see Table 3). Corresponding ratings showed an at least average mean value in all genders and age groups (with one exception). In contrast, participants rather denied that YouTubers show responsible handling of their role model function. Moreover, females perceived a greater role model function of YouTubers than males did. Regarding age effects, the under-16-year-old group perceived a weaker role model function but better responsible handling than the 16- to 20year-old group. Finally, the perceived importance of YouTubers as role models and their similarity to music and movie stars significantly increased from age group to age group.

\section{Learning and Influenceability (RQ3a, RQ3b)}

Regarding the importance of YTPS-videos for participants' learning about politics and societal topics (RQ3a), we found below-average ratings independently of age and gender, all $t \mathrm{~s} \leq-2.72, p \mathrm{~s} \leq .009, d \mathrm{~s} \leq-.354$ (adjusted significance level $p=.0083$ ). However, YTPS-videos were rated as more important for learning by male participants $(M=2.56, S D=0.91)$ than by female participants $(M=2.33, S D=0.93), F(1$, $556)=7.28, p=.007, \eta_{\mathrm{p}}{ }^{2}=.013$. There were no further main or interaction effects, all $F_{\mathrm{s}} \leq 2.59, p \mathrm{~s} \geq .076, \eta_{\mathrm{p}}{ }^{2} \leq .009$.

Regarding the perceived influenceability by YTPS-videos (RQ3b), Fig. 1 shows participants' assessments of how strongly they let themselves be influenced and to what extent other people of the same age, younger people, and older people are presumed to be influenceable by such videos. Males and females in all age groups consistently perceived their own and older people's influenceability as below average, whereas younger persons' influenceability was consistently rated as above average. In contrast, the three age groups perceived a different influenceability of other people of the same age (i.e., other peers): The under-16-year-old males, the over-20-yearsold males, and 16- to 20-years-old females rated them as averagely influenceable. The over-20-year-old females reported a below-average rating, and the other two groups an aboveaverage rating. Next, we extended our usual two-way ANOVA to a 3 (age group) $\times 2$ (gender) $\times 4$ (valuation subject) ANOVA with valuation subject as a within-subject factor (self vs. other people of same age vs. younger people vs. older people) and perceived influenceability by YTPS-videos as dependent variable. We also calculated Bonferroni-adjusted pairwise comparisons in case of a significant main effect of valuation subject. We found a main effect of valuation subject, $F(2.64,1459.50)=894.23, p<.001, \eta_{\mathrm{p}}{ }^{2}=.618$ : The participants perceived themselves and older people as less influenceable than younger people and people of the same age. Besides, the influenceability of younger people was rated higher than that of people of the same age. We found a main effect of age group, $F(2,552)=5.32, p=.005, \eta_{\mathrm{p}}{ }^{2}=.019$ : The over-20year-old group estimated people's influenceability lower than the other two age groups. However, we also found an interaction between age group and valuation subject, $F(5.29$, 
Fig. 1 Participants' assessments of the influenceability of themselves, other peers, younger persons, and older persons by YTPS-videos. Vertical lines indicate the standard error of the mean. Asterisks indicate the results of one-sample $t$-tests against the scales' midpoint of 3 . ${ }^{1}$ The respective test result is not significant when applying the Bonferroni-adjusted significance level $(p=.002)$ to the set of $t$-tests

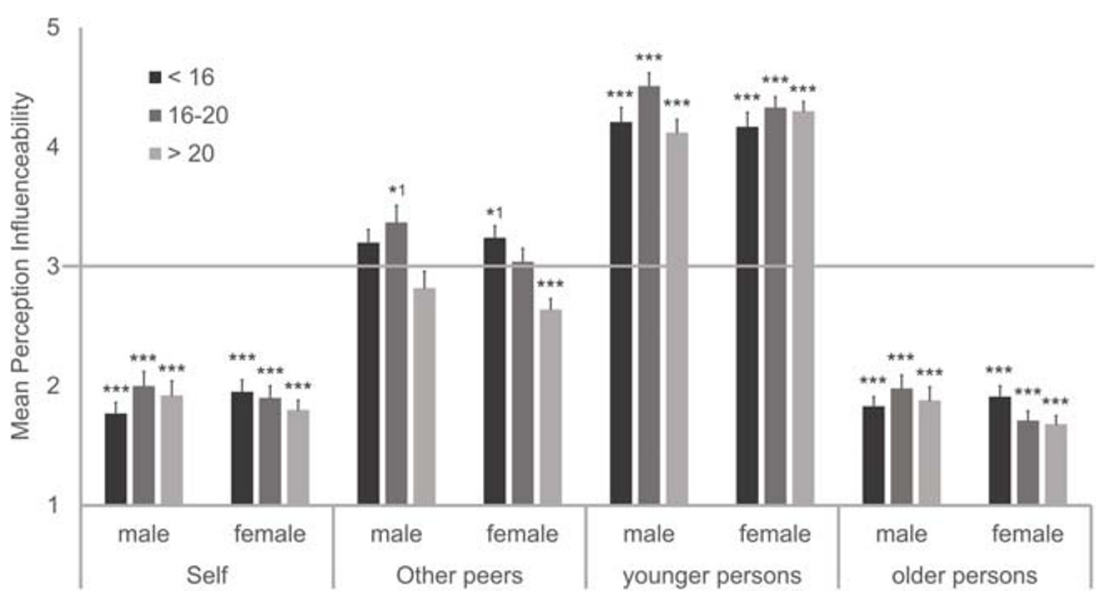

$1459.50)=4.17, p<.001, \eta_{\mathrm{p}}{ }^{2}=.015$ : The effect of age group was only significant regarding the evaluation of other people of the same age, $F(2,556)=14.50, p<.001, \eta_{\mathrm{p}}{ }^{2}=.050$.

\section{Communication about YTPS-Videos (RQ4a, RQ4b)}

Concerning the participants' communication partners regarding the content of YTPS-videos (RQ4a), we found that participants of both genders and all age groups reported a belowaverage communication frequency with the presented communication partners, but preferred communicating with their friends instead of teachers, see Table 4. The ANOVAs revealed that males communicated more often with friends and classmates. Furthermore, the over-20-year-old group talked with their family members (including parents, aunts, uncles, grandparents) less often about the content of YTPSvideos than the younger age groups did. We additionally found two interactions between age group and gender: In the 16- to 20-year-old group, males communicated more often with their family than females did, $t(153)=2.35, p=.020$, $d=.390$. Furthermore, males communicated more often with their friends than females in the under-16-year-old group, $t(192.31)=2.36, p=.019, d=.328$, and in the 16-20-yearold group, $t(154)=22.19, p=.030, d=.362$.

In terms of the communication channels the participants use when communicating about the content of YTPS-videos (RQ4b), face-to-face communication was the most preferred modus, see Table 4 . All other channels were used relatively rarely. We found several main effects of gender and age group, but no interactions: Males, compared to females, communicated more frequently via YouTube comments, Facebook's news feed, E-Mail, Skype, and face-to-face. The over-20-year-old group used the Facebook's news feed and phone calls more often, but they communicated via Snapchat and Instagram less frequently about the content of YTPSvideos than the other two age groups. Also, the over-20year-old group communicated via E-Mail and Skype more often than the under-16-year-old group. Additionally, the
16- to 20-year-old group talked face-to-face more frequently compared to the other two age groups.

\section{Discussion}

The present study examined the role of YTPS-videos and YouTubers for young people's opinion formation on political and societal topics.

First, we found that young people reported a generally high YouTube usage intensity and a moderate watching frequency of YTPS-videos (RQ1), indicating that YouTube was an important information source in our sample. While general usage intensity was exceptionally high in the 16 - to 20 -year-old group, the over-20-year-old group less often visited YouTube channels but showed an even higher watching frequency of YTPS-videos compared to the under-16-year-old group. Notably, males reported an overall higher YouTube consumption.

Second, participants' responses reflected some critical perceptions of YTPS-videos and YouTubers, as already found in other subject areas (Martínez \& Olsson, 2019). Though the liking of YTPS-videos was relatively high, participants perceived them as only moderately credible (RQ2a). The over20 -year-old evaluated YTPS-videos as less credible and reported a lower appreciation of YouTubers' own opinion than both younger age groups. Further, they stated a lower liking of YTPS-videos than the 16- to 20-year-old group. These results indicate that young people's critical perception of YTPSvideos increases with age, while there was no effect of gender. Interestingly, credibility evaluation was positively correlated with viewing frequency in the oldest age group. Also, we found positive correlations between the credibility evaluation and the appreciation of the presentation of YouTubers' own opinion in all groups, but the highest correlations were found in the over-20-year-old group. Hence, credibility seems to become a more critical factor for consumption frequency 
and media acceptance with increasing age, supporting previous findings (Nelson \& Kim, 2020).

Third, participants moderately agreed to all characteristics of YTPS-videos and their producers as being indicative of fake news (RQ2b). This result also considerably varied between age groups. Again, the over-20-year-old group appeared to be more critical than the under-16-year-old group regarding the citation of information sources, YouTubers' attempt to be a role model, and the extent of personal advice given by the YouTubers. Gender differences were negligible. Very young people may struggle with the evaluation of online news as already observed in previous studies (Flanagin \& Metzger, 2000; McGrew, Breakstone, Ortega, Smith, \& Wineburg, 2018; Metzger et al., 2003; Nee, 2019). This would also underline the importance of a tool for reconnaissance to improve media criticism in the context of fake news on YouTube, as it was suggested by McGrew et al. (2018). Young people could also refer to other characteristics than those addressed in the present study and previously reported by Hugger et al. (2019), for example design, graphics, and multimedia content (Agosto, 2002; Shenton \& Dixon, 2004; Wobbrock, Hsu, Burger, \& Magee, 2019).

Fourth, we found a very pronounced difference in participants' evaluation of YTPS-videos compared to traditional TV news (RQ2c). The latter was perceived as more objective, facts-oriented, informative, credible, neutral, reputable, and professional but also more boring, independently of participants' age and gender. In contrast, YTPS-videos were generally seen as more entertaining, emotional, funny, exciting, modern, motivating, but also more subjective, opinion-oriented, and manipulative than traditional TV news, independently of age and gender. This result pattern is compatible with the findings of Elvestad, Phillips, and Feuerstein (2017) who surveyed students from Israel, Norway, and the United Kingdom, all reporting higher trust in traditional news media than in social media. Such results may reflect a critical perception or even scepticism of YTPS-news on the one hand. On the other hand, it also indicates a rather uncritical perception of TV news and only a little critical reflection on traditional journalism processes. With respect to age, we found that the over-20year-old group showed a more balanced comparison between traditional TV news and YTPS-news. Surprisingly, female participants, compared to male participants, rated TV news as more objective, facts-oriented, informative, exciting, credible, neutral, reputable, and compact, and they rated YTPSvideos as more subjective, opinion-oriented, emotional, and manipulative. We may speculate that this result pattern either shows a higher capacity for media criticism in young females or a greater orientation towards supposedly valid media standards. Future research should disentangle these possibilities in more detail.

Fifth, participants ascribed YouTubers an important role model function and a high awareness of their influence, independently of gender and age (RQ2d). In addition, they criticised YouTubers' rather irresponsible handling of their role model function. The perceived importance of YouTubers as role models and their similarity to music and movie stars increased from age group to age group. De Veirman et al. (2019) already stressed the importance of influencers as relatable and approachable role models as children are willing to build (parasocial) relationships and to identify with them. Thus, this is a critical mixture. On the one hand, YouTubers are vital role models, but in contrast to traditional celebrities, the perceived distance is less pronounced, increasing their potential effects on the young audience. As found by Schouten et al. (2020), recipients tend to identify more with, feel more similar to, and trust influencers more compared to celebrities. Interestingly, participants of the present study, independently of age and gender, also assumed that YouTubers would be only moderately aware of their role model function. To sum up, the participants seem to (partially) understand and critically reflect on the YouTubers' roles and potential effects.

Sixth, the participants reported that YTPS-videos are relatively unimportant for their learning about politics and societal topics, but YTPS-videos were rated as more important for learning by male participants than by female participants (RQ3a). Therefore, purposeful and intentional use of YouTube for the acquisition of political and societal knowledge appears not prevalent. It would be interesting to see whether there are nonetheless learning gains by consumption. Indeed, unintentional learning has already been shown in experimental settings (e.g., Schmidt \& De Houwer, 2012). Also, are there differences in knowledge between males versus females? It would be an interesting direction for future research to combine YouTube consumption with objective measures of knowledge performance.

Seventh, young people consistently perceived themselves as less influenceable than younger people and same-aged people, and as influenceable as older people (RQ3b). Young people seem to perceive themselves as relatively resistant to the influences of YTPS-video content, although they are aware of the YTPS-videos' manipulative potential. This finding is supported by previous research focusing on influencer marketing on YouTube, which came to similar results (Coates, Hardman, Halford, Christiansen, \& Boyland, 2020). Also, the fact that participants perceived themselves as significantly less influenceable by mass media than younger and same-aged people is consistent with the well-known third-person effect (Davison, 1983) and previous findings in the context of social media and online news (Houston et al., 2011; Schweisberger et al., 2014). Consequently, they overestimate their resistance or underestimate that of other people.

Finally, we found that participants of both genders and all age groups reported a below-average communication frequency about the content of YTPS-videos with usual 
communication partners, but preferred communicating with their friends instead of teachers (RQ4a). That result is crucial as it indicates that a critical debriefing and analysis of the consumed content elude the formal educational contexts in school, which might have a corrective effect on political education processes. A possible explanation could be that the roles of parents and teachers concerning online activities decrease with age during adolescence, while the one of peers increases. This circumstance has already been shown by previous studies in the context of teenagers' engagement in online risks (Shin \& Lwin, 2017). Another reason could be that parents and teachers do not provide the appropriate room for discussions about YTPS-videos. At the same time, face-toface communication was the most preferred modus when communicating about the content of YTPS-videos (RQ4b). This result may be interpreted as promising because this form of communication provides an appropriate basis for discussing complex issues. In contrast, some communication technologies suffer from information transfer capacities (Daft, Lengel, \& Trevino, 1987) and may additionally be associated with critical data security issues.

\section{Practical Implications and Future Research}

The results allow us to conclude about media education of young people at school. Young people do not seem to discuss the topics of YTPS-videos with their teachers in school often. One possible question which arises in this context is whether school plays a role at all when it comes to the consumption of, discussion about, and critical reflection on YTPS-videos. It could be difficult for schools and teachers to address such private and niched consumption adequately. Media psychological research may help to disentangle the situational and personal obstacles counteracting the desirable discussion and comparison of individual standpoints. Importantly, social media platforms such as YouTube and the topic of fake news have already been included in course syllabi and are nothing new to be inserted into classroom teaching. However, the critical question is how these topics can be addressed in formal education in an appropriate and target group-oriented manner. The present results indicate that formats focusing on peer-to-peer communication might be the key to success. It is also unclear to what extent teachers actually make this content the subject of instruction and whether students are willing to talk about such content. Content of YTPS-videos might be considered sensitive and thus requires specific methods facilitating the discussion of sensitive topics with young people (e.g., Hoppe, Wells, Morrison, Gillmore, \& Wilsdon, 1995). It should be noted that nowadays school curricula already include media literacy as a learning aim, but the approaches vary a lot between different schools and countries (Zhang, Zhang, \& Wang, 2020). For example, in Germany, where the present study took place, no centralised, standardised policy for media literacy exist. Thus, the critical question is how specific content of new media, such as YTPSvideos, can be integrated into formal education settings so that this creates added value for students. Psychological research can help to systematically examine the effects of media-based learning formats, as recently shown in the context of commercial video games (e.g., Rüth \& Kaspar, 2020).

Besides, the implications of the third-person effect could be addressed pedagogically. From a user-oriented perspective, an underestimation of one's own influenceability appears more problematic than an overestimation of other people's influenceability by mass media. Because persuasive effects of influencers on young people are evident (e.g., De Veirman et al., 2019; Dehghani, Niaki, Ramezani, \& Sali, 2016; Sokolova \& Kefi, 2020), such an underestimation could increase young people's vulnerability to persuasive attempts of YTPSvideos. One possible countermeasure could be the discussion with teachers in class. Teachers could help to pinpoint the relevance of political and societal topics presented in the YTPSvideos for the young people. As Schweisberger et al. (2014) showed, the perceived effects of news stories on oneself are higher for relevant news compared to non-relevant ones. Additionally, the authors found that the third-person effect seems to be smaller for high-relevant topics. Thus, pinpointing the relevance of news for young people could raise awareness for the persuasive effects of YTPS-video content. Furthermore, teachers could explain the effects of social media and influencers on young people as well as the implications of the third-personeffect. Such educational measures might significantly improve young people's media literacy. However, we have to emphasise that our results do not provide any information on whether the third-person effect reflects young people's underestimation of their influenceability. Davison (1983) alternatively stated, "that people will tend to overestimate the influence that mass communications have on the attitudes and behavior of others" (p. 3). In accordance, Sun, Pan, and Shen (2008) showed that third-person effects in terms of the perceived self-other asymmetry are larger when others are perceived as particularly vulnerable. This finding could indicate that the third-person effect instead represents an overestimation of the influenceability of others. Also, only small and inconsistent relations between the third-person effect and behavioural intentions were found (Xu \& Gonzenbach, 2008). As a result, the question arises whether the third-person effect found in the present study is of practical relevance at all. This question should be clarified by future research before addressing the third-person effect pedagogically in school. Alarming claims of effects that do not exist should be avoided in the context of media literacy education.

Our findings also form the basis for future quantitative studies. We provide first quantitative results about young people's use and critical reflection of YTPS-videos. These results may facilitate deriving and testing concrete hypotheses, but our results also open new directions for further research topics. Indeed, research on the role of YTPS-videos on young people's opinion formation 
regarding political and societal topics has been widely neglected so far but appears very important given the high relevance of this medium for the young generation. Besides, we found first and strong evidence for gender and age differences in the use and evaluation of YTPS-videos. Future research should consider such differential effects and may also include personality traits. Also, what are the antecedents and consequences of such gender effects regarding media consumption, education, and political orientation? Temporal changes in media literacy are also conceivable. Up to now, no longitudinal studies are addressing this. Such studies could clarify whether the age differences found in the present study represent truly longitudinal changes or indicate cohort effects. Finally, the findings also deliver first implications and relevant aspects for the development and testing of educational programmes. Based on our results, such educational programmes could address, for example, methods to verify YTPS-news and online information, persuasive attempts of such videos, as well as the critical reflection of users' behaviour and attitudes regarding YTPS-videos to foster media literacy.

\section{Limitations and Prospects for Future Research}

There are some more limitations of the present study which should be considered when interpreting the results above. First, we developed our research topics mainly based on research from other subject areas and several older studies due to a lack of thematically more specific research. As a consequence, this study was mainly explorative. In addition, we were also not able to use established and validated instruments. As YouTube and YTPS-videos as a platform for information gathering become more and more important, it would be desirable to develop validated multi-item scales to assess the specific aspects examined here. In this way, the constructs, their relationships, their factorial structure, and their construct validity can be researched in more detail. The present study may provide the first basis for such projects. It should also be noted that few results become not significant when applying a more conservative, Bonferroni-adjusted significant level.

Moreover, we investigated pupils from a high school and university students with a relatively high educational background in Germany. Besides, as participation in this study was voluntary and the online version was distributed via specific social media groups, the degree of a potential sample bias cannot be finally estimated. In each case, the sample was rather homogenous. There is evidence that the ability for media criticism varies between schools: For example, $\mathrm{Hu}$ and Kurokami (2017) found differences in media literacy between pupils of an urban and of a rural middle school in China. Pupils of the urban middle school showed, among other things, higher critical thinking skills. The authors mainly attributed this to the missing curriculum and opportunities to explore and learn the appropriate media use in rural middle schools. A further aspect of this context of learning opportunities is that media criticism seems to be taught mainly within higher education. Schmidt (2013) examined how media literacy is addressed in different levels of the educational system in the US. The author found that media literacy is most often addressed in higher education like university and college compared to earlier school forms like Middle or High School. Consequently, the results in the present study could be different when investigating young people who attend a school form at another educational level. Further, as media literacy issues are mainly addressed in higher education, the higher media literacy skills of our older age group could simply reflect a higher number of passed learning opportunities. Consequently, future research on media literacy regarding YTPS-videos has to take school form and educational background into account.

Finally, in contrast to other accounts (e.g., Martínez \& Olsson, 2019), the present study did not focus on the perception of a specific YouTuber and their videos. We did not specify the source of the YTPS-videos consumed by the participants. As the reported number of subscribed channels showed a median of 10 in the present study, participants' responses refer to a rather general impression of YouTubers and YTPS-videos. The present data thus provide a generalised picture that lays the foundation for more differentiated studies in the future. For example, as Lewis (2020) showed, some YouTubers rather represent more extreme and reactionary political standpoints by sensationalising mainstream news. As a result, the perception and evaluation of YouTubers and YTPS-videos could vary, depending on how the YouTubers' political orientations are perceived and how they match with the orientation of the individual consumer. Indeed, perceived similarity with an influencer can have positive effects on consumers' trust in influencer postings and on behavioural intentions (e.g., Lou \& Yuan, 2019). Additionally, the political orientation could be more pronounced with increasing age. As a consequence, political orientations of both the consumer and the YouTuber become more relevant with increasing age. Also, the specific attributes of the videos might act as a moderator on perception effects. In the present study, we purposefully covered a vast range of YTPS-videos that was neither limited to specific political or societal topics nor to a specific video format. In general, it is conceivable that the perception of YTPS-videos varies across topics, genres, contents, stylistic choices, viewer groups, report types, and commercial ties of the YouTubers. For example, we found that commercialisation seems to be a relevant aspect for young people when evaluating YTPS-videos. This could in turn influence the persuasiveness of the video content. Future research should disentangle the contribution of specific attributes of YTPS-videos on young people's perception.

To conclude, the present study was one first attempt to bridge a vital research gap regarding young people's analytic-critical perception and evaluation of YouTubers and YTPS-videos. We found significant age and gender effects and a complex result pattern highlighting implications for both future research and educational measures. This study provides 
a substantial basis for a better understanding of how young people perceive and evaluate media content, media producers, and their role in the media consumption process.

Authors' Contributions (CRediT Roles) LG, KUW and KK acquired the funding; LG, KUH and KK administered the project; DZ, CN, LG, KUH and KK designed the study; DZ, CN, LG, LMB, TN and KK collected the data; DZ and KK analysed the data; DZ and KK wrote the manuscript; KK supervised the study; CN, LG, KUH, LMB and TN approved submission.

Funding Open Access funding enabled and organized by Projekt DEAL. This work was partly funded by the Federal State Government of NorthRhine Westphalia (NRW, Germany) via the Grimme Research College (Project: YouTuber and Peers/2017-2018).

Data Availability The data that support the findings of this study, as well as the used materials, are available from the corresponding author upon reasonable request.

\section{Compliance with Ethical Standards}

Conflict of Interest On behalf of all authors, the corresponding author states that there is no conflict of interest.

Ethics Approval All procedures performed in the study were in accordance with the ethical guidelines of the German Psychological Society (DGPs) and with the 1964 Helsinki declaration. According to the guidelines of the German Research Association, no ethical approval was needed because the research did not pose any threats or risks to the respondents, it was not associated with high physical or emotional stress, and the respondents were informed about the objectives of the study (http://www. dfg.de/foerderung/faq/geistes_sozialwissenschaften/index.html).

Consent to Participate/for Publication Written informed consent to participate in this study was provided by the students or (if younger than 18 years) by their legal guardians/next of kin, and all students voluntarily participated in this study.

\section{Code Availability Not applicable.}

Open Access This article is licensed under a Creative Commons Attribution 4.0 International License, which permits use, sharing, adaptation, distribution and reproduction in any medium or format, as long as you give appropriate credit to the original author(s) and the source, provide a link to the Creative Commons licence, and indicate if changes were made. The images or other third party material in this article are included in the article's Creative Commons licence, unless indicated otherwise in a credit line to the material. If material is not included in the article's Creative Commons licence and your intended use is not permitted by statutory regulation or exceeds the permitted use, you will need to obtain permission directly from the copyright holder. To view a copy of this licence, visit http://creativecommons.org/licenses/by/4.0/.

\section{References}

Agosto, D. E. (2002). A model of young people's decision-making in using the web. Library \& Information Science Research, 24(4), 311-341. https://doi.org/10.1016/S0740-8188(02)00131-7.
Balaban, D., \& Mustățea, M. (2019). Users' perspective on the credibility of social media influencers in Romania and Germany. Romanian Journal of Communication and Public Relations, 21(1), 31-46.

Banning, S. A., \& Sweetser, K. D. (2007). How much do they think it affects them and whom do they believe? Comparing the thirdperson effect and credibility of blogs and traditional media. Communication Quarterly, 55(4), 451-466. https://doi.org/10.1080/ 01463370701665114.

Coates, A. E., Hardman, C. A., Halford, J. C. G., Christiansen, P., \& Boyland, E. J. (2020). "It's just addictive people that make addictive videos": Children's understanding of and attitudes towards influencer Marketing of Food and Beverages by YouTube video bloggers. International Journal of Environmental Research and Public Health, 17(2), article 449. https://doi.org/10.3390/ ijerph17020449.

Daft, R. L., Lengel, R. H., \& Trevino, L. K. (1987). Message equivocality, media selection, and manager performance: Implications for information systems. MIS Quarterly, 11, 355-366. https://doi.org/ $10.2307 / 248682$.

Davison, W. P. (1983). The third-person effect in communication. Public Opinion Quarterly, 47(1), 1-15. https://doi.org/10.1086/268763.

De Jans, S., Hudders, L., \& Cauberghe, V. (2018). Adolescents' selfreported level of dispositional advertising literacy: How do adolescents resist advertising in the current commercial media environment? Young Consumers, 19(4), 402-420. https://doi.org/10.1108/ YC-02-2018-00782.

De Veirman, M., Hudders, L., \& Nelson, M. R. (2019). What is influencer marketing and how does it target children? A review and direction for future research. Frontiers in Psychology, 10, article 2685. https://doi.org/10.3389/fpsyg.2019.02685.

Dehghani, M., Niaki, M. K., Ramezani, I., \& Sali, R. (2016). Evaluating the influence of YouTube advertising for attraction of young customers. Computers in Human Behavior, 59, 165-172. https://doi. org/10.1016/j.chb.2016.01.037.

Djafarova, E., \& Rushworth, C. (2017). Exploring the credibility of online celebrities' Instagram profiles in influencing the purchase decisions of young female users. Computers in Human Behavior, $68,1-$ 7. https://doi.org/10.1016/j.chb.2016.11.009.

Duong, H., Nguyen, L., \& Vu, H. (2020). With whom do consumers interact?: Effects of online comments and perceived similarity on source credibility, content credibility, and personal risk perception. Journal of Social Marketing, 10(1), 18-37. https://doi.org/10.1108/ JSOCM-02-2019-0023.

Dyosi, N., \& Hattingh, M. (2018). Understanding the extent of and factors involved in the use of YouTube as an informal learning tool by 11-to 13-year-old children. In T. T. Wu, Y. M. Huang, R. Shadiev, L. Lin, \& A. Starčič (Eds.), International conference on innovative technologies and learning (pp. 351-361). Springer: Cham. https:// doi.org/10.1007/978-3-319-99737-7_38.

Elvestad, E., Phillips, A., \& Feuerstein, M. (2017). Can trust in traditional news media explain cross-national differences in news exposure of young people online? A comparative study of Israel, Norway and the United Kingdom. Digital Journalism, 6(2), 216-235. https://doi. org/10.1080/21670811.2017.1332484.

Flanagin, A. J., \& Metzger, M. J. (2000). Perceptions of internet information credibility. Journalism \& Mass Communication Quarterly, 77(3), 515-540. https://doi.org/10.1177/107769900007700304.

Gangadharbatla, H., Bright, L. F., \& Logan, K. (2014). Social media and news gathering: Tapping into the millennial mindset. The Journal of Social Media in Society, 3(1).

GDPR (2016). Regulations. Regulation (EU) 2016/679 of the European Parliament and of the council of 27 April 2016 on the protection of natural persons with regard to the processing of personal data and on the free movement of such data, and repealing directive 95/46/EC (general data protection regulation). Retrieved June 5, 2020, from 
https://eur-lex.europa.eu/legal-content/EN/TXT/PDF/?uri=CELEX: 32016R0679

Grinberg, N., Joseph, K., Friedland, L., Swire-Thompson, B., \& Lazer, D. (2019). Fake news on twitter during the 2016 US presidential election. Science, 363(6425), 374-378. https://doi.org/10.1126/science. aau2706.

Guess, A., Nagler, J., \& Tucker, J. (2019). Less than you think: Prevalence and predictors of fake news dissemination on Facebook. Science Advances, 5(1), article eaau4586. https://doi. org/10.1126/sciadv.aau4586.

Hoppe, M. J., Wells, E. A., Morrison, D. M., Gillmore, M. R., \& Wilsdon, A. (1995). Using focus groups to discuss sensitive topics with children. Evaluation Review, 19(1), 102-114. https://doi.org/ 10.1177/0193841X9501900105.

Houston, J. B., Hansen, G. J., \& Nisbett, G. S. (2011). Influence of user comments on perceptions of media bias and third-person effect in online news. Electronic News, 5(2), 79-92. https://doi.org/10.1177/ 1931243111407618.

Hu, X., \& Kurokami, H. (2017). A comparison of media literacy in urban and rural middle school students. International Journal for Educational Media and Technology, 11(1), 132-140. Retrieved June 22, 2020, from http://jaems.jp/contents/icomej/vol11/R03 Hu.pdf

Hudders, L., \& Cauberghe, V. (2018). The mediating role of advertising literacy and the moderating influence of parental mediation on how children of different ages react to brand placements. Journal of Consumer Behaviour, 17(2), 197-210. https://doi.org/10.1002/cb. 1704.

Hudders, L., Cauberghe, V., \& Panic, K. (2016). How advertising literacy training affect children's responses to television commercials versus advergames. International Journal of Advertising, 35(6), 909-931. https://doi.org/10.1080/02650487.2015.1090045.

Hugger, K., Braun, L. M., Noll, C., Nowak, T., Gräßer, L., Zimmermann, D., \& Kaspar, K. (2019). Zwischen Authentizität und Inszenierung: Zur medienkritischen Einschätzung informationsorientierter YouTuber*innen-Videos durch Jugendliche [Between authenticity and staging: On the media-critical evaluation of informationoriented YouTuber videos by young people]. In: F. von Gross, \& R. Röllecke (Hrsg.), Instagram und YouTube der (Pre-)Teens Inspiration, Beeinflussung, Teilhabe [Instagram and YouTube of (pre-)teens - inspiration, influence, participation] (pp. 29-36). Munich: kopaed.

Jafari, M., \& Ansari-Pour, N. (2019). Why, when and how to adjust your P values? Cell Journal (Yakhteh), 20(4), 604-607. https://doi.org/ 10.22074/cellj.2019.5992.

Jiménez, A. G., García, B. C., \& de Ayala, M. C. L. (2016). Adolescents and Youtube: Creation, participation and consumption. Prisma Social: revista de investigación social, 1, 60-89.

Kaspar, K., \& Müller-Jensen, M. (2019). Information seeking behavior on Facebook: The role of censorship endorsement and personality. Current Psychology, online first, 1-12. https://doi.org/10.1007/ s12144-019-00316-8.

Khan, M. L. (2017). Social media engagement: What motivates user participation and consumption on YouTube? Computers in Human Behavior, 66, 236-247. https://doi.org/10.1016/j.chb.2016.09.024.

Krasnova, H., Veltri, N. F., Eling, N., \& Buxmann, P. (2017). Why men and women continue to use social networking sites: The role of gender differences. The Journal of Strategic Information Systems, 26(4), 261-284. https://doi.org/10.1016/j.jsis.2017.01.004.

Lee, S., \& Lee, D. K. (2018). What is the proper way to apply the multiple comparison test? Korean Journal of Anesthesiology, 71(5), 353360. https://doi.org/10.4097/kja.d.18.00242.

Lewis, R. (2020). "This is what the news Won't show you": YouTube creators and the reactionary politics of micro-celebrity. Television \& New Media, 21(2), 201-217. https://doi.org/10.1177/1527476419879919.
Lou, C., \& Yuan, S. (2019). Influencer marketing: How message value and credibility affect consumer rust of branded content on social media. Journal of Interactive Advertising, 19(1), 58-73. https:// doi.org/10.1080/15252019.2018.1533501.

Lowry, P. B., Wilson, D. W., \& Haig, W. L. (2013). A picture is worth a thousand words: Source credibility theory applied to logo and website design for heightened credibility and consumer trust. International Journal of Human-Computer Interaction, 30(1), 6393. https://doi.org/10.1080/10447318.2013.839899.

Martínez, C., \& Olsson, T. (2019). Making sense of YouTubers: How Swedish children construct and negotiate the YouTuber Misslisibell as a girl celebrity. Journal of Children and Media, 13(1), 36-52. https://doi.org/10.1080/17482798.2018.1517656.

McGrew, S., Breakstone, J., Ortega, T., Smith, M., \& Wineburg, S. (2018). Can students evaluate online sources? Learning from assessments of civic online reasoning. Theory \& Research in Social Education, 46(2), 165-193. https://doi.org/10.1080/00933104. 2017.1416320 .

Medienpädagogischer Forschungsverband Südwest (MPFS) (2020). JIM-Studie 2019. Basisuntersuchung zum Medienumgang 12- bis 19-Jähriger [JIM Study 2019: A Basic study on media use among 12- to 19-year-olds]. Retrieved June 22, 2020, from https://www. mpfs.de/fileadmin/files/Studien/JIM/2019/JIM_2019.pdf

Metzger, M. J., Flanagin, A. J., \& Zwarun, L. (2003). College student web use, perceptions of information credibility, and verification behavior. Computers \& Education, 41(3), 271-290. https://doi.org/10. 1016/S0360-1315(03)00049-6.

Metzger, M. J., Flanagin, A. J., \& Medders, R. B. (2010). Social and heuristic approaches to credibility evaluation online. Journal of Communication, 60(3), 413-439. https://doi.org/10.1111/j.14602466.2010.01488.x.

Moghavvemi, S., Sulaiman, A., Jaafar, N. I., \& Kasem, N. (2018). Social media as a complementary learning tool for teaching and learning: The case of youtube. The International Journal of Management Education, 16(1), 37-42. https://doi.org/10.1016/j.ijme.2017.12. 001.

Nee, R. C. (2019). Youthquakes in a post-truth era: Exploring Social media news use and information verification actions among global teens and young adults. Journalism \& Mass Communication Educator, 74(2), 171-184. https://doi.org/10.1177/1077695818825215.

Nelson, J. L., \& Kim, S. J. (2020). Improve trust, increase loyalty? Analyzing the Relationship Between News Credibility and Consumption. Journalism Practice, 1-18. https://doi.org/10.1080/ 17512786.2020.1719874.

Pires, F., Masanet M.-J. \& Scolari C. A. (2019). What are teens doing with YouTube? Practices, uses and metaphors of the most popular audio-visual platform. Information, Communication \& Society, 117. https://doi.org/10.1080/1369118X.2019.1672766.

Questback, G. H. (2017). EFS survey, version summer 2017. Cologne: Questback GmbH.

Rozendaal, E., Buijzen, M., \& Valkenburg, P. (2010). Comparing children's and adults' cognitive advertising competences in the Netherlands. Journal of Children and Media, 4(1), 77-89. https:// doi.org/10.1080/17482790903407333.

Rüth, M., \& Kaspar, K. (2020). Exergames in formal school teaching: A pre-post longitudinal field study on the effects of a dance game on motor learning, physical enjoyment, and learning motivation. Entertainment Computing, 35, 100372. https://doi.org/10.1016/j. entcom.2020.100372.

Schmidt, H. C. (2013). Media literacy education from kindergarten to college: A comparison of how media literacy is addressed across the educational system. Journal of Media Literacy Education, 5(1). Retrieved from https://digitalcommons.uri.edu/jmle/vol5/iss1/3

Schmidt, J. R., \& De Houwer, J. (2012). Adding the goal to learn strengthens learning in an unintentional learning task. 
Psychonomic Bulletin \& Review, 19(4), 723-728. https://doi.org/10. 3758/s13423-012-0255-5.

Schmidt, J. H., Hölig, S., Merten, L., \& Hasebrink, U. (2017). Nachrichtennutzung und Meinungsbildung in Zeiten sozialer Medien [news usage and opinion formation in times of social media]. Informatik-Spektrum, 40(4), 358-361. https://doi.org/10.1007/ s00287-017-1057-y.

Schouten, A. P., Janssen, L., \& Verspaget, M. (2020). Celebrity vs. influencer endorsements in advertising: The role of identification, credibility, and product-endorser fit. International Journal of Advertising, 39(2), 258-281. https://doi.org/10.1080/02650487. 2019.1634898 .

Schweisberger, V., Billinson, J., \& Chock, T. M. (2014). Facebook, the third-person effect, and the differential impact hypothesis. Journal of Computer-Mediated Communication, 19(3), 403-413. https://doi. org/10.1111/jcc4.12061.

Shenton, A. K., \& Dixon, P. (2004). Issues arising from youngsters' information-seeking behavior. Library \& Information Science Research, 26(2), 177-200. https://doi.org/10.1016/j.lisr.2003.12. 003.

Shin, W., \& Lwin, M. O. (2017). How does "talking about the internet with others" affect teenagers' experience of online risks? The role of active mediation by parents, peers, and school teachers. New Media \& Society, 19(7), 1109-1126. https://doi.org/10.1177/ 2F1461444815626612.

Shu, K., Sliva, A., Wang, S., Tang, J., \& Liu, H. (2017). Fake news detection on social media: A data mining perspective. $A C M$ SIGKDD Explorations Newsletter, 19(1), 22-36. https://doi.org/ $10.1145 / 3137597.3137600$.

Sokolova, K., \& Kefi, H. (2020). Instagram and YouTube bloggers promote it, why should I buy? How credibility and parasocial interaction influence purchase intentions. Journal of Retailing and Consumer Services, 53. https://doi.org/10.1016/j.jretconser.2019. 01.011

Statista (2020). Most popular social networks worldwide as of July 2020, ranked by number of active users. https://www.statista.com/ statistics/272014/global-social-networks-ranked-by-number-ofusers/. Accessed 13 Nov 2020

Sun, Y., Pan, Z., \& Shen, L. (2008). Understanding the third-person perception: Evidence from a meta-analysis. Journal of Communication, 58(2), 280-300. https://doi.org/10.1111/j.14602466.2008.00385.x.

Todd, P. R., \& Melancon, J. (2018). Gender and live-streaming: Source credibility and motivation. Journal of Research in Interactive Marketing, 12(1), 79-93. https://doi.org/10.1108/JRIM-05-20170035.

van Dam, S., \& van Reijmersdal, E. (2019). Insights in adolescents' advertising literacy, perceptions and responses regarding sponsored influencer videos and disclosures. Cyberpsychology: Journal of Psychosocial Research on Cyberspace, 13(2), article 2. https://doi. org/10.5817/CP2019-2-2.

Van Cleemput, K. (2010). "I'll see you on IM, text, or call you": A social network approach of adolescents' use of communication media. Bulletin of Science, Technology \& Society, 30(2), 75-85. https:// doi.org/10.1177/0270467610363143.

Vermeulen, A., Vandebosch, H., \& Heirman, W. (2018). Shall I call, text, post it online or just tell it face-to-face? How and why Flemish adolescents choose to share their emotions on-or offline. Journal of Children and Media, 12(1), 81-97. https://doi.org/10.1080/ 17482798.2017.1386580.

Waddell, T. F. (2018a). What does the crowd think? How online comments and popularity metrics affect news credibility and issue importance. New Media \& Society, 20(8), 3068-3083. https://doi.org/ $10.1177 / 1461444817742905$.

Waddell, T. F. (2018b). This tweet brought to you by a journalist: How comment gatekeeping influences online news credibility. Electronic News, 12(4), 218-234. https://doi.org/10.1177/1931243117739946.

Warner-Søderholm, G., Bertsch, A., Sawe, E., Lee, D., Wolfe, T., Meyer, J., Engel, J., \& Fatilua, U. N. (2018). Who trusts social media? Computers in Human Behavior, 81, 303-315. https://doi.org/10. 1016/j.chb.2017.12.026.

Wobbrock, J. O., Hsu, A. K., Burger, M. A., \& Magee, M. J. (2019), Isolating the effects of web page visual appearance on the perceived credibility of online news among college students. In Proceedings of the 30th ACM Conference on Hypertext and Social Media (pp. 191200). Association for Computing Machinery. https://doi.org/10. $1145 / 3342220.3343663$

Xiao, M., Wang, R., \& Chan-Olmsted, S. (2018). Factors affecting YouTube influencer marketing credibility: A heuristic-systematic model. Journal of Media Business Studies, 15(3), 188-213. https:// doi.org/10.1080/16522354.2018.1501146.

Xu, J., \& Gonzenbach, W. J. (2008). Does a perceptual discrepancy lead to action? A meta-analysis of the behavioral component of the thirdperson effect. International Journal of Public Opinion Research, 20(3), 375-385. https://doi.org/10.1093/ijpor/edn031.

Zhang, L., Zhang, H., \& Wang, K. (2020). Media literacy education and curriculum integration: A literature review. International Journal of Contemporary Education, 3(1), 55-64. https://doi.org/10.11114/ ijce.v3i1.4769.

Zilka, G. C. (2018). Medium preferences of children and adolescents for content distributed by the media. Interchange, 49(4), 457-476. https://doi.org/10.1007/s10780-018-9337-2.

Publisher's Note Springer Nature remains neutral with regard to jurisdictional claims in published maps and institutional affiliations. 